\title{
ACTUALITÉ DE LA PHILOSOPHIE DE FERDINAND GONSETH
}

\author{
Gilles COHEN-TANNOUDJI
}

RÉSUMÉ : Les progrès les plus récents de la physique contemporaine, en particulier la convergence de la physique des particules et de la cosmologie en une authentique cosmogonie scientifique, avivent les enjeux philosophiques de l'entreprise scientifique, mais les rapports conflictuels entre physique et philosophie ne s'en trouvent pas apaisés ; un fossé semble même en train de se creuser entre science et philosophie. L'œuvre du philosophe suisse Ferdinand Gonseth qui s'est attaché à élaborer une philosophie qui soit et qui puisse rester au niveau de la connaissance scientifique peut fournir une aide particulièrement adéquate à ceux qui voudraient contribuer à combler ce fossé.

MotS-CLÉS : Ferdinand Gonseth, physique, philosophie, cosmologie, cosmogonie.

ABSTRACT : The most recent progresses of contemporary physics, in particular the merging of particle physics and cosmology into a genuine scientific cosmogony revive the philosophical stakes of the scientific enterprise, but the adversarial relationship between physics and philosophy is not alleviated; a ditch seems even to be deepening hollow between science and philosophy. The work of the Swiss philosopher Ferdinand Gonseth who endeavored to work out a philosophy which is and which can remain at the level of scientific knowledge can provide a particularly adequate help to those who would like to contribute to filling this ditch.

KEYWORDS : Ferdinand Gonseth, physics, philosophy, cosmology, cosmogony. 
ZuSAMMENFASSUNG : Die jüngsten Fortschritte der heutigen Physik, insbesondere die Konvergenz der Teilchenphysik und der Kosmologie zu einer genuinen Kosmogonie, lassen die philosophischen Aspekte der wissenschaftlichen Unternehmung wieder aufleben. Aber die konfliktreichen Beziehungen zwischen Physik und Philosophie kommen nicht zur Ruhe, vielmehr scheint sich geradezu ein Graben zwischen Wissenschaft und Philosophie aufzutun. Das Werk des schweizerischen Philosophen Ferdinand Gonseth, der sich das Erarbeiten einer Philosophie vorgenommen hatte, die auf dem Niveau des wissenschaftlichen Wissens bleiben sollte und könnte, kann ein besonders angemessenes Hilfsmittel für diejenigen bereitstellen, die zum Zuschütten dieses Grabens beitragen möchten.

STICHWÖRTER : Ferdinand Gonseth, Physik, Philosophie, Kosmologie, Kosmogonie.

Gilles CoHEn-TanNoudi, né en 1938, est conseiller scientifique auprès du directeur des Sciences de la matière du Commissariat à l'énergie atomique. Il est retraité de cet organisme, dans lequel il a fait toute sa carrière comme physicien théoricien dans le domaine de la physique des particules. Actuellement, il prépare une thèse de philosophie, sous la direction de Dominique Lecourt, sur la philosophie de Ferdinand Gonseth.

Adresse : CEA Saclay, 91191 Gif-sur-Yvette Cedex.

Courrier électronique : cohen@dsmdir.cea.fr ; gicotan@club-internet.fr 
Le 14 décembre 1900, Max Planck présentait, devant la Société allemande de physique, le travail dans lequel il expliquait le spectre universel de rayonnement du corps noir au moyen de la théorie des quanta d'énergie. Cette date est considérée comme la date de naissance de la physique des quanta, qui a révolutionné la physique, et au travers elle l'ensemble des sciences et des technologies. La Suisse a joué et joue un rôle très important dans ce mouvement de la science. En effet, c'est en Suisse, qu'un jeune et obscur employé des brevets à Berne, nommé Albert Einstein, devait, dès 1905, presque en même temps qu'il jetait les bases de la théorie de la relativité, corroborer les travaux de Planck et les élargir en émettant l'hypothèse révolutionnaire du caractère discontinu du champ électromagnétique. D'autre part, c'est en Suisse que devait exercer son activité le grand physicien, dont nous avons célébré le centenaire, Wolfgang Pauli. Enfin, tout le monde sait que c'est en Suisse qu'est implanté le CERN (Conseil européen pour la recherche nucléaire), capitale mondiale de la physique des particules, où l'on a découvert les bosons intermédiaires, et où se préparent, avec le LHC (Large Hadron Collider Project), les plus grandes expériences scientifiques jamais réalisées par l'humanité.

C'est à l'œuvre du philosophe suisse Ferdinand Gonseth, disparu en 1975, que je consacre la thèse de philosophie que je prépare actuellement sous la direction de Dominique Lecourt.

Ma carrière de physicien théoricien au CEA (Commissariat à l'énergie atomique) débuta en octobre 1962 à Saclay. La discipline que j'ai choisie, la physique des particules, prenait alors son essor. Rétrospectivement, il apparaît que ce fut une grande chance, puisque j'ai pu être un témoin et, pour une toute petite part, un acteur d'une des plus formidables mutations qui se soient opérées dans la connaissance humaine. Depuis les années 1960, sont intervenues en effet trois avancées majeures :

1) l'émergence de ce que l'on appelle le modèle standard de la physique des particules, qui décrit avec une extraordinaire précision l'ensemble des données expérimentales concernant les constituants élémentaires de la matière et les interactions qualifiées de fondamentales dans lesquelles ces constituants sont impliqués ;

2) l'émergence du modèle standard de la cosmologie, appelé modèle du big bang, selon lequel notre univers est en expansion et en refroidissement depuis une phase primordiale de température et de densité infinies ;

3) la convergence de ces deux modèles standard, leur coopération qui leur confère une dimension temporelle, cosmogonique.

Qui, mieux que Gonseth, pourrait mettre en évidence les immenses enjeux philosophiques de ces avancées scientifiques ? C'est en effet lui qui a préfacé le livre dans lequel le chanoine Georges Lemaître exposait en 1946 son « hypothèse ${ }^{1}$ de l'atome primitif » qui devait devenir le fameux modèle du big bang. Dans cette préface, Gonseth souligne que

1. Dans son livre consacré à la vie et à l'œuvre de Georges Lemaître, LAMBERT, 2000, souligne l'influence philosophique profonde qu'a eue Ferdinand Gonseth sur le père du big bang et il note «qu'il n'est pas impossible que le philosophe suisse ait proposé ce titre qui semble tellement bien rejoindre sa "philosophie ouverte" ». 
« la portée d'une hypothèse cosmogonique dépasse d'une manière incommensurable la sphère de l'utile immédiat et touche à la nécessité. Comme la voûte du ciel surplombe et enveloppe la terre de toutes parts, une certaine doctrine cosmogonique domine et enveloppe la conscience de l'homme, la conscience de tous les hommes ».

\section{Puis il conclut :}

«Quel que soit l'avenir de l'hypothèse de l'atome primitif, on peut dire sans exagération que la grandiose perspective qu'elle dessine dans le monde phénoménal se réfléchit en une grandiose perspective sur le plan philosophique ${ }^{2}$. »

\section{PHYSIQUE ET PHILOSOPHIE, DES RAPPORTS COMPLEXES}

\section{Mécanisme, atomisme et positivisme}

À la fin du XIX ${ }^{\mathrm{e}}$ siècle, la physique, l'archétype des sciences de la nature, était parvenue à un stade de grand épanouissement, qui faisait dire à certains qu'elle était en voie d'achèvement. Jusqu'à la naissance de la science moderne, avec les travaux fondateurs de Galilée, Newton, Leibniz et Descartes, la pensée du monde et de notre rapport au monde était à peu près exclusivement l'affaire des philosophes, ou, plus précisément, l'affaire des grands systèmes philosophiques, comme la conception des quatre éléments ou la conception atomiste. La théorie de Newton, qui réalisait l'unification des phénomènes qui se passent sur terre et de ceux qui concernent le mouvement des astres célestes, marque une rupture majeure. Comme le dit Gonseth,

« la mécanique de Newton donne une cause au mouvement des planètes sur leurs orbites : l'attraction mutuelle des planètes et du Soleil. Toute l'explication des mouvements des planètes sur la voûte céleste, à travers les constellations des étoiles fixes, vient donc se fonder dans la matière et dans les lois auxquelles obéit la matière. Pour prévoir tout l'avenir et pour reconstituer tout le passé du système solaire, il suffisait en principe de connaître la distribution des masses matérielles dans l'espace et leurs vitesses en un instant déterminé. Le monde physique se refermait sur lui-même et se donnait à lui-même la loi fondamentale. Un monde matérialiste avait pris naissance ${ }^{3} »$.

Il va sans dire qu'une telle révolution scientifique, dont le développement de la cosmologie moderne est en quelque sorte le prolongement, ne pouvait pas ne pas avoir de profondes répercussions sur l'ensemble de la philosophie. Il devenait urgent de repenser les catégories fondamentales de matière, de temps et d'espace. C'est essentiellement à cette tâche que s'est attelé Kant :

«La théorie kantienne de la connaissance est un essai de purification de la situation de connaissance propre à la science newtonienne, un essai de désigner ce qui doit être nécessairement assuré d'avance pour que cette science puisse être ce qu'elle était. Le

2. LEMAÎTRE, 1946, p. 23.

3. GONSETH, 1953, p. 38. 
rôle que le temps et l'espace jouent dans la science newtonienne se trouve consacré par leur élévation au rang d'intuitions a priori dans la doctrine kantienne ${ }^{4}$. »

Ainsi voyait-on un système philosophique se construire dans la perspective de s'adapter à une grande avancée scientifique, voire de tenter de la récupérer ${ }^{5}$.

Mais c'est surtout sur le plan strictement scientifique que la percée des travaux de Newton ouvrait de très grandes perspectives. La méthode expérimentale, inaugurée par Galilée, et la mathématisation mise en œuvre par Newton ouvraient la voie à un programme ambitieux, le programme mécaniste (ne devrait-on pas dire mécaniciste ?), qui vise à ramener l'ensemble de la physique aux lois du mouvement des objets matériels dans l'espace et dans le temps. La mécanique se sépare alors en deux parties : d'une part, l'art de fabriquer des machines, à l'origine de la révolution industrielle du XIX siècle, et d'autre part la mécanique rationnelle, qui consiste à poser et à résoudre les équations du mouvement des divers objets matériels dont le monde est constitué. Il est tout à fait remarquable que ce programme non seulement reste encore d'actualité de nos jours, mais encore qu'il se soit épanoui dans la cosmogonie scientifique que nous avons évoquée plus haut. C'est d'ailleurs pour lever les difficultés rencontrées dans sa mise en œuvre qu'ont été accomplies les grandes remises en cause de la révolution scientifique $\mathrm{du} \mathrm{XX}^{\mathrm{e}}$ siècle.

Grâce aux travaux de Lagrange, Euler, Hamilton et Jacobi, la mécanique rationnelle atteint un degré inégalé d'efficacité. La conception de l'espace s'affine : on passe de l'espace de la géométrie élémentaire à trois dimensions, à des espaces abstraits de représentation du mouvement des objets matériels, comme l'espace de configuration et l'espace de phase, dont le nombre de dimensions est proportionnel au nombre de degrés de liberté du système matériel à l'étude. La compréhension de l'articulation entre invariances, symétries et lois de conservation, permettait de dégager des critères de réalité, en faisant jouer un rôle fondamental au concept d'énergie.

Comme il fallait s'y attendre, la philosophie s'est adaptée pour intégrer les développements rapides de la science. Cette adaptation est bien résumée par le positivisme d'Auguste Comte. Cette doctrine distingue trois stades dans le développement des connaissances humaines : le stade théologique ou fictif, le stade métaphysique ou abstrait et, enfin, le stade scientifique ou positif. Mais cette doctrine a suivi la pente naturelle de toute philosophie, celle qui consiste à s'ériger en système. Ayant procédé à une classification de toutes les sciences, le positivisme tend à énoncer un critère absolu de réalité : n'est réel que ce que la science, parvenue à un stade achevé, nous dévoile. C'est au nom de ce critère que les physiciens d'inspiration positiviste comme Wilhelm Ostwald ou Ernst Mach se sont opposés avec acharnement aux travaux de James Clerk Maxwell et Ludwig Boltzmann qui, avec la théorie cinétique de la matière et la thermodynamique statistique, s'efforçaient de rendre compatibles l'hypothèse atomique et la mécanique rationnelle. Maxwell et Boltzmann acceptaient l'hypothèse atomique, c'est-à-dire celle d'une limite à la divisibilité de la matière, et ils essayaient d'appliquer

4. GONSETH, 1953, p. 42.

5. Dans le chapitre intitulé « Kant et les quanta » du livre consacré au Siècle des quanta, LeCourT, 2003, montre que ce que les physiciens fondateurs de la mécanique quantique appellent « la physique classique » n'est en fait que la physique newtonienne, profondément remaniée par Kant. 
la mécanique rationnelle au mouvement des constituants irréductibles de la matière, les atomes ou molécules. À la fin du XIX ${ }^{\mathrm{e}}$ siècle, on peut dire que grâce à leurs travaux, la conception atomiste, qui pendant près de vingt-cinq siècles était restée une simple hypothèse philosophique, commençait à se transformer en une authentique théorie scientifique ; il devenait possible d'unifier l'ensemble des descriptions phénoménologiques et macroscopiques de la matière, et de les intégrer au programme de la mécanique rationnelle ; la chimie pouvait se détacher définitivement des méandres de l'alchimie et devenir une science à part entière capable de nous éclairer sur les processus qui se développent au sein de la matière. Malheureusement, il a fallu attendre encore quelques années pour que l'existence effective des atomes puisse être expérimentalement mise en évidence, un laps de temps qui a été mis à profit par les philosophes et physiciens d'inspiration positiviste pour rejeter ce qui s'annonçait comme les prémices d'une grande révolution scientifique et conceptuelle.

\section{La révolution des quanta et de la relativité}

Cela nous amène à la période qui va de 1895 à la fin des années 1920, au cours de laquelle s'est jouée la révolution scientifique qui a donné naissance à la science contemporaine. Pour apprécier la difficulté des problèmes posés par la découverte des quanta, le mieux est peut-être de citer l'auteur même de cette découverte, Max Planck, qui s'exprime ainsi dans son Autobiographie scientifique :

«Le rôle joué par cette constante nouvelle dans le déroulement uniformément régulier des processus physiques restait encore une question tout à fait obscure. J'essayai donc immédiatement de rattacher d'une manière quelconque le quantum élémentaire d'action $h$ au cadre de la physique classique. [...] L'échec de toutes mes tentatives pour sauter l'obstacle me rendit bientôt évident le rôle fondamental joué par le quantum élémentaire d'action dans la physique atomique, et que son apparition ouvrait une ère nouvelle dans les sciences de la nature. Car elle annonçait l'avènement de quelque chose d'entièrement inattendu et elle était destinée à bouleverser les bases mêmes de la pensée physique, qui depuis la découverte du calcul infinitésimal s'appuyaient sur l'idée que toutes les relations causales sont continues ${ }^{6}$. »

Outre la remise en cause du dogme du continu dans les relations causales, la constante de Planck traduit une nouvelle limitation fondamentale qui s'impose à tout sujet connaissant dans son rapport cognitif avec la nature : à moins de faire une expérience durant un temps infini, on ne peut pas observer une particule microscopique sans lui transférer un tant soit peu d'énergie, et si, réciproquement, on veut observer un objet microscopique avec une grande précision spatio-temporelle, on doit lui communiquer une énergie d'autant plus élevée que la précision souhaitée est élevée. Il devient dès lors impossible de faire abstraction des conditions de l'observation du monde microscopique. Ainsi, ce n'est pas seulement la causalité qui est mise en cause par le quantum d'action, mais c'est l'objectivité elle-même, l'autre pilier de toute l'entreprise scientifique

6. Planck, 1960, p. 93-94. 
qu'il va falloir redéfinir. Pour lever ces difficultés, il a été nécessaire de modifier de fond en comble le formalisme de la physique.

D'autres remises en cause fondamentales ont été impliquées par la théorie de la relativité : matérialité de la lumière, conception profondément modifiée de l'espace et du temps, critique de notions jusqu'alors considérées comme évidentes comme la simultanéité, autant de questionnements qui, à l'instar de la remise en cause des critères classiques d'objectivité impliquée par la découverte des quanta, n'allaient pas manquer de provoquer de profonds débats proprement philosophiques.

\section{Physique et philosophie, un fossé qui se creuse?}

Ce qu'il convient de noter d'emblée à propos de ces débats philosophiques appelés par le développement de la physique, c'est leur nouveauté radicale et l'impossibilité dans laquelle se sont trouvés les différents systèmes philosophiques alors établis d'y apporter quelque contribution utile. Le positivisme lui-même, qui se prétendait être le système philosophique le plus adéquat au développement de la science, se trouva en échec : dès la fin de la première décennie du siècle, il avait fallu se rendre à l'évidence, les atomes, dont le positivisme niait l'existence, étaient une réalité expérimentalement tangible. Comme le dit Jean Perrin, à la fin de son livre de 1913, intitulé Les Atomes :

«La théorie atomique a triomphé. Encore nombreux naguère, ses adversaires enfin conquis renoncent l'un après l'autre aux défiances qui, longtemps, furent légitimes et sans doute utiles. C'est au sujet d'autres idées que se poursuivra désormais le conflit des instincts de prudence et d'audace dont l'équilibre est nécessaire au lent progrès de la science humaine ${ }^{7}$. »

Ce n'est pas mon propos ici de m'étendre sur le formidable essor des sciences qui a suivi la révolution du début $\mathrm{du} \mathrm{Xx}^{\mathrm{e}}$ siècle. Tout le monde sait que le monde observable s'étend considérablement aussi bien en direction de l'infiniment petit des particules élémentaires que de l'infiniment grand de l'astrophysique et de la cosmologie. Comme nous l'avons évoqué en introduction, le rapprochement de ces deux disciplines débouche sur une authentique cosmogonie selon laquelle l'univers nous apparaît comme en devenir depuis une phase indifférenciée jusqu'à l'état dans lequel il se laisse observer, à la suite de toute une série de transitions de phases au cours desquelles les symétries se brisent, les interactions se différencient, certaines particules acquièrent leurs masses, de nouvelles structures ou états de la matière émergent. Ces progrès sont concomitants d'autres faisant intervenir un troisième infini, l'infiniment complexe, et ayant ouvert à la science l'accès à une immense diversité de structures, de phénomènes ou de situations, et d'applications technologiques. Il faut noter, dans ce domaine, le rôle primordial du concept d'information qui débouche aujourd'hui sur les nouvelles technologies de l'information et de la communication, en train de faire muter nos sociétés.

C'est surtout le problème philosophique rencontré par les fondateurs de la physique contemporaine qui sera évoqué ici. Ce problème est, tout compte fait, le problème fondamental de toute la philosophie, celui du rapport de la matière à la pensée, ou

7. PERRIN, 1991, p. 284-285. 
encore celui de l'adéquation du rationnel au réel. Il faut bien reconnaître en effet que la physique quantique qui se construisait et, dans une moindre mesure, la théorie de la relativité avaient de quoi dérouter ceux qui ne voulaient pas se contenter de considérer la science comme un simple ensemble de recettes. D'après le consensus mécaniste qui prévalait alors, on pensait que l'objectivité de la réalité physique ne posait aucun véritable problème : la réduction de la réalité en deux sous-ensembles, celui qui dépend de l'observateur et celui qui n'en dépend pas, ne posait aucune difficulté de principe. Il semblait possible de séparer nettement l'objectif du subjectif ; la mécanique classique ignorait le sujet de la connaissance. En physique classique, il est toujours possible, en principe, de faire abstraction des conditions de l'observation ; à la rigueur, ces conditions sont présentes, en pratique, dans les marges d'incertitudes ou «barres d'erreurs », avec lesquelles sont présentés les résultats des expériences. Mais avec la physique quantique, on est confronté à l'impossibilité, de principe, de faire abstraction des conditions de l'observation; le mouvement de la connaissance serait-il alors contraint de revenir en arrière, du stade scientifique au stade métaphysique ? Faudrait-il renoncer à la recherche de l'objectivité inhérente à toute démarche scientifique ? Les fantastiques progrès scientifiques permis par la physique quantique démontrent à l'évidence qu'il n'en est rien. Encore fallait-il résoudre de façon satisfaisante le problème philosophique qui était posé. Les physiciens du début du siècle ont pris la mesure du défi qu'ils avaient à relever, d'autant plus qu'ils savaient ne pouvoir compter sur aucun des systèmes philosophiques existants. Ils se sont attelés à la tâche dans un intense bouillonnement de débats et de controverses sans concessions, comme ceux qui ont opposé pendant de nombreuses années Bohr et Einstein. À la fin des années 1920, un certain consensus était atteint sur ce que l'on a appelé l'interprétation de l'école de Copenhague. Selon cette école, il convenait de distinguer au sein de la physique quantique le formalisme mathématique et l'interprétation de ce formalisme, c'est-à-dire la façon dont ce formalisme est capable de rendre compte du résultat des expériences ou des observations. Autant le formalisme semblait rigoureux, efficace et infaillible, autant l'interprétation semblait comporter des zones d'ombre et même des failles qui fournissaient l'occasion de controverses persistantes, comme celles qui ont perduré autour du « chat de Schrödinger » ou du paradoxe d'Einstein, Podolsky et Rosen. Ce décalage entre formalisme et interprétation a encore accru l'incompréhension de la plupart des philosophes face à un développement scientifique particulièrement ardu pour des non-spécialistes. L'efficacité un peu mystérieuse du formalisme, comparée à la relative faiblesse de l'interprétation, a alimenté les courants du néopositivisme ${ }^{8}$ et de l'empirisme logique, voire un retour au relativisme. Il faut bien reconnaître que les développements formels de plus en plus techniques qui ont suivi cette période n'ont pas contribué à combler ce fossé. Tant et si bien que nous sommes actuellement dans une phase de blocage du dialogue entre philosophes et physiciens, comme en témoigne le chapitre intitulé «Contre la philosophie » dans le livre récent du prix Nobel de physique Stephen Weinberg ${ }^{9}$, ou

8. Sans compter que certains physiciens de l'école de Copenhague se sont laissé aller à des positions franchement positivistes, comme lorsqu'ils ont voulu dissuader Hideki Yukawa de proposer une théorie impliquant l'existence d'une nouvelle particule, le méson.

9. WeINBERG, 1996. 
bien ce que l'on a appelé « l'affaire Sokal ${ }^{10}$ », du nom de ce physicien qui s'en est pris vivement au relativisme prôné par certains philosophes comme Bruno Latour :

«Latour prétend ensuite s'adresser à la sociologie des sciences, mais son exposé est confus : il mélange allègrement ontologie et épistémologie, et s'attaque à des thèses que personne ne soutiendrait. "Au lieu de reconnaître une science à l' exactitude absolue de son savoir, on la reconnait à la qualité de l'expérience collective qu'elle montre" - mais qui de nos jours prétendrait que la science fournit des "exactitudes absolues" ? La mécanique newtonienne décrit le mouvement des planètes (et beaucoup d'autres choses) avec une précision extraordinaire - et ceci est un fait objectif - mais elle est néanmoins incorrecte. La mécanique quantique et la relativité générale sont de meilleures approximations de la vérité - et ceci également est un fait objectif - mais elles aussi, étant incompatibles, seront sans doute un jour supplantées par une théorie (encore inexistante) de la gravitation quantique. Tout scientifique sait bien que nos connaissances sont toujours partielles et révisables - ce qui ne les empêche pas d'être objectives. De la même manière, Latour réduit le relativisme à une banale "capacité à changer de point de vue", comme si celle-ci n'était pas depuis longtemps une des caractéristiques par excellence de l'attitude scientifique ${ }^{11}$. »

Mais cette attitude hostile vis-à-vis de la philosophie venant des physiciens peut faire craindre une aggravation des formes de rejet de la science qui se manifestent actuellement dans nos sociétés, par exemple sous forme de désaffection des jeunes pour les études scientifiques. Avec la perspective de cosmogonie scientifique que nous avons évoquée plus haut, la plus technique des disciplines scientifiques, la physique, s'aventure sur un terrain auquel aucune religion ou croyance ne peut être indifférente. La philosophie, dans toutes ses dimensions (ontologique, gnoséologique et axiologique), sera-t-elle à la hauteur de sa tâche de combattre symétriquement le scientisme et l'obscurantisme ? C'est, encore une fois, à Gonseth que l'on peut emprunter l'exorde de l'admirable texte concluant La Métaphysique et l'ouverture à l'expérience : après avoir montré les menaces extrêmes que ferait peser le « désenracinement philosophique de la superélite scientifique », il expose la tâche qui attend la philosophie face à la science,

« facteur de puissance et ferment d'évolution. [...] Mais sans aller jusque-là, sans aller jusqu'à la menace et la contrainte mortelle, exercée sur les esprits par l'intermédiaire de la menace de la contrainte exercée sur les corps, l'évolution dont nous voyons dès maintenant les effets mettra la philosophie devant une tâche hérissée de difficultés. Encore une fois, je ne parle pas de la philosophie qui se mettrait à l'abri et à l'écart, dans la tour d'un système bien construit, mais fermé au défi que l'expérience pourrait lui jeter. Dans le monde tel qu'il est, dans l'histoire telle qu'elle se fait, une philosophie de ce genre laissera fatalement l'essentiel de sa mission lui échapper. Mais quel pourrait être le rôle d'une philosophie qui accepterait pleinement sa fonction au sein même de l'éternel changement, celle de mettre et de remettre constamment à découvert les valeurs à promouvoir? Je ne lui vois pas d'autre destin que de se lier à la science, non pour en être la servante, mais pour lui rester toujours égale. Non pour la suivre en tout, mais pour l'accompagner partout, pour se mesurer partout avec elle et ne jamais lui

10. BRICMONT et SOKAL, 1997.

11. SoKal, 1997. 
céder le terrain en toute propriété. Nul ne peut prévoir les péripéties de ce dialogue, où chacun lutterait pour les autres en luttant pour soi-même.

«On peut espérer que la philosophie y trouverait quelque force et la science quelque sagesse.

« À celui qui me dira "tout ce qui ne vise pas l'absolu vise trop bas", je répondrai "à qui veut trop, tout est repris" $12 »$.

C'est parce que je souhaite contribuer, autant que faire se peut, à combler le menaçant fossé qui se creuse entre science et philosophie que j'ai entrepris de confronter la philosophie de Gonseth aux développements de la physique contemporaine. Mathématicien de formation et de profession, Gonseth (1890-1975) a été, à mon avis, l'un des seuls philosophes du $\mathrm{XX}^{\mathrm{e}}$ siècle à avoir compris la science de son siècle et à avoir proposé une philosophie digne de cette science. Comme il le dit dans Mon itinéraire philosophique ${ }^{13}$, son option fondamentale consiste à privilégier la recherche par rapport aux systèmes et, au sein de la recherche, à privilégier la recherche scientifique.

Tout en continuant à enseigner les mathématiques, il a suivi avec une très grande attention les développements de la physique théorique, et y a même contribué à quelques reprises. En 1928, il signe avec Gustave Juvet un article dans Helvetica Physica Acta intitulé «Sur la relativité à cinq dimensions et sur une interprétation de l'équation de Schrödinger ${ }^{14} »$. Dans Mon itinéraire philosophique, Gonseth se plaint que cet article soit quelque peu passé inaperçu, mais, quand on sait l'importance qu'ont prise à l'heure actuelle les idées de dimensions supplémentaires de l'espace pour l'unification de la relativité générale et de la mécanique quantique, on ne peut qu'admirer la perspicacité de Gonseth et Juvet qui, dès 1928, s'y étaient intéressés, précisément dans cette perspective.

Tout au long de son œuvre, Gonseth s'est attaché à comprendre, de l'intérieur, la stratégie de la recherche scientifique face au problème de l'adéquation du rationnel au réel. Ainsi, pouvons-nous voir comment il caractérise le mouvement de la science dans «Motivation et structure d'une philosophie ouverte », chapitre d'introduction aux entretiens de Rome (repris dans Philosophie néo-scolastique et philosophie ouverte) :

«Celui qui a vécu, dans le doute d'abord, les premiers temps de la théorie de la relativité, et, plus tard, le développement de la théorie des quanta, qui les a vues se confirmer d'un œil et d'un esprit presque incrédules, celui-là sait désormais que les audaces de la pensée scientifique n'ont rien d'arbitraire, et qu'elles témoignent au contraire d'une sûreté qui serait inconcevable, si l'on ne savait de quelle chaîne ininterrompue d'essais et d'efforts elle a été payée. Il y a dans la science telle qu'elle se fait, telle qu'elle s'avance, telle qu'elle s'aventure et telle qu'elle se corrige, une si évidente correspondance au réel que le doute systématique n'est plus permis à un esprit informé et sincère ${ }^{15}$. »

Il constate, à partir d'une analyse minutieuse de l'histoire de la géométrie ${ }^{16}$, que la stratégie de la recherche scientifique est plutôt une stratégie d'engagement (plus tard,

12. GONSETH, 1973a, p. 291.

13. GONSETH, 1994.

14. Gonseth et JUVEt, 1928.

15. GONSETH, 1973b, p. 16.

16. Gonseth, 1949. 
dans Mon itinéraire philosophique, il utilise un terme qu'il trouve plus « idoine », celui de cheminement) qu'une stratégie de fondement ${ }^{17}$, et il en tire une option philosophique fondamentale : l'ouverture à l'expérience. Pour la philosophie, l'avancée de la science est une expérience qu'elle ne peut pas ne pas prendre en compte si elle ne veut pas être marginalisée. D'autre part, au plan strictement philosophique, la stratégie de fondement est condamnée par la pluralité des systèmes et par leur échec face à l'antinomie du commencement. Une stratégie philosophique d'engagement lève cette antinomie et permet une approche dialectique du problème de l'adéquation du rationnel au réel. C'est ainsi que le concept clé d'horizon de réalité permet d'élucider nombre des difficultés rencontrées lors du développement de la physique quantique :

« À première vue, les deux parties de l'expression "le monde propre" ne s'accordent guère : la première met l'accent sur la réalité extérieure, sur la réalité du monde naturel ; la seconde évoque au contraire l'idée que la forme sous laquelle l'homme conçoit le monde n'est que la transcription de la structure même de ses facultés de connaître : la première partie est réaliste, la seconde idéaliste. C'est d'ailleurs aussi le cas de l'expression "horizon de réalité" dont la première partie relativise et subjectivise la seconde. Un tel mariage d'expressions tirant dans des directions aussi nettement opposées est-il admissible ? Je le crois nécessaire, pour éviter de tomber dans l'une ou dans l'autre de deux erreurs elles-mêmes opposées. La première consisterait à dire que notre conception du monde n'est qu'une empreinte, en nous, du monde extérieur existant en dehors de nous avec tous ses caractères objectifs ; la seconde serait d'affirmer, de façon en quelque sorte symétrique, que la réalité telle que nous la concevons n'est qu'une production de notre esprit, qu'elle n'est que la projection de notre structure mentale. L'idée de "l'horizon de réalité" se détourne de l'une et de l'autre de ces interprétations trop simples, trop extrêmes. Cette idée est médiatrice entre celle de l'autonomie de l'esprit et de la prédétermination inconditionnelle de la réalité du monde. Elle ne fait que répondre à l'expérience de la connaissance scientifique sur laquelle nous insistions déjà tout au début : au dévoilement progressif de la réalité. Elle a été conçue pour recueillir cette expérience et lui donner sa forme conceptuelle ${ }^{18}$. »

C'est dans le numéro consacré à la notion de complémentarité, de la revue Dialectica dont est extraite cette citation que s'étaient exprimés les pères fondateurs de la théorie quantique tels Wolfgang Pauli, Niels Bohr, Werner Heisenberg, Louis de Broglie et Albert Einstein. Gonseth, directeur de la revue, concluait ce « fascicule publié sous la direction de Wolfgang Pauli » par une «Remarque sur l'idée de complémentarité ». Dans son éditorial, Pauli avait exprimé l'accord des physiciens de l'école de Copenhague avec les vues philosophiques de Gonseth. La coopération, voire la connivence des deux hommes pour la réalisation de cet ouvrage essentiel pour l'histoire de la mécanique quantique apparaît incontestable.

Si Gonseth s'est toujours trouvé en phase avec les développements les plus avancés de la science, c'est parce qu'il avait mesuré l'enjeu de la compréhension de ce qu'est la méthodologie scientifique. Cette préoccupation méthodologique traverse toute son œuvre. On la retrouve explicitement dans ses livres principaux, qu'ils soient consacrés

17. Gonseth, 1968.

18. Gonseth, 1948. 
à l'espace ${ }^{19}$, ou au temps ${ }^{20}$ et dans la quasi-totalité de ses articles. Parti des mathématiques et des sciences de la nature, il s'est convaincu que la méthodologie « des quatre phases et des quatre principes ${ }^{21}$ » était aussi pleinement à l'œuvre dans les sciences humaines, des sciences vers lesquelles il s'est tourné vers la fin de sa vie, en s'attaquant à des problèmes de philosophie générale, comme celui de la morale ou celui de la foi.

Le $\mathrm{XX}^{\mathrm{e}}$ siècle a vu naître, se déployer et s'épanouir une révolution scientifique d'une extraordinaire ampleur du point de vue de ses conséquences et d'une exceptionnelle profondeur à l'égard des remises en cause qu'elle implique. Une telle révolution scientifique appelle une révolution philosophique au moins aussi profonde. Je ne sais pas si tous les philosophes ont pris conscience du défi qui est ainsi lancé à la philosophie. En tout cas, il semblerait que Gonseth l'ait bien compris, comme il le dit dans ce texte, extrait de Mon itinéraire philosophique, où il analyse (à la troisième personne) son livre sur le problème du temps:

«L'œuvre philosophique de Ferdinand Gonseth est toute dominée par la triple intention que voici :

«Fonder une philosophie qui soit et qui puisse rester au niveau de la connaissance scientifique.

«Dégager cette philosophie non pas de principes posés a priori comme nécessaires, mais de la pratique et du progrès même de la recherche.

«L'engager à titre d'épreuve dans toutes les perspectives déjà ouvertes. »

Après avoir explicité son intention, il souligne l'ampleur de la tâche qu'il s'est donnée :

« Il n'y avait qu'une preuve à donner de l'existence d'une telle philosophie : lui conférer l'existence en la faisant de toutes pièces. C'est cette démonstration-là que M. Gonseth prétend avoir pour le moins esquissée dans son dernier ouvrage ${ }^{22}$. »

Voilà pourquoi je suis profondément convaincu que l'on peut trouver chez Ferdinand Gonseth des moyens très efficaces pour tenter de combler le fossé qui se creuse entre la science et la philosophie.

Gilles COHEN-TANNOUdJI

(octobre 2004).

19. GONSETH, 1949.

20. GonsETh, 1964. Alors que l'approche du problème de l'espace s'appuyait sur le développement d'une science mathématique, la géométrie, l'approche du problème du temps aborde deux autres problématiques, celle d'abord du langage (comme 1'a si bien démontré EMERY, 1998) et celle de l'instrumentation scientifique, en liaison avec la mesure du temps, si importante en Suisse, la patrie de l'industrie horlogère, mais aussi pays hôte du CERN !

21. EMERY, 1985.

22. Gonseth, 1994, p. 187. 


\section{LISTE DES RÉFÉRENCES}

BRICMONT (Jean) et SoKAL (Alan), 1997, Impostures intellectuelles, Paris, Odile Jacob.

EMERY (Éric), 1985, Ferdinand Gonseth. Pour une philosophie «dialectique » ouverte à l'expérience, Lausanne, L'Âge d'Homme.

EMERY (É.), 1998, Temps et musique, Lausanne, L’Âge d'Homme.

GonsETH (Ferdinand) et JuVET (Gustave), 1928, « Sur la relativité à cinq dimensions et sur une interprétation de l'équation de Schrödinger », Helvetica Physica Acta, vol. I, fasc. 6, p. 421-436.

Gonseth (Ferdinand), 1948, «Quelques remarques sur l'idée de complémentarité », Dialectica, vol. II, fasc. 3-4, p. 413-420.

Gonseth (F.), 1949, La Géométrie et le problème de l'espace, Neuchâtel, Le Griffon.

Gonseth (F.), 1953, «Philosophie et sciences », conférence présentée au symposium de la Société suisse de philosophie, Berne, 10-11 oct. 1953, Studia Philosophica, Separatum, vol. XIII, p. 36-44.

Gonseth (F.), 1964, Le Problème du temps. Essai sur la méthodologie de la recherche, Neuchâtel, Le Griffon.

Gonseth (F.), 1968, « Stratégie de fondement et stratégie d'engagement », Dialectica, vol. XXII, fasc. 3-4, p. 171-186.

Gonseth (F.), 1973a, La Métaphysique et l'ouverture à l'expérience, Lausanne, L'Âge d'Homme.

Gonseth (F.), 1973b, Philosophie néo-scolastique et philosophie ouverte, Lausanne, L'Âge d'Homme.

Gonseth (F.), 1994, Mon itinéraire philosophique, présentation de François Bonsack, Vevey, L'Aire.

LAMBERT (Dominique), 2000, Un atome d'univers, Bruxelles, Racines.

LATOUR (Bruno), 1997, "Y a-t-il une science après la Guerre froide ? », Le Monde, 18 janv., p. 17.

Lecourt (Dominique), 2003, « Kant et les quanta », in Crozon (Michel) et SACQuin (Yves), éd., Un siècle de quanta, Les Ulis, EDP Sciences, p. 149-158.

LEMAîtRE (Georges), 1946, L'Hypothèse de l'atome primitif, préf. de Ferdinand GonsETH, Neuchâtel, Le Griffon.

Perrin (Jean), 1991, Les Atomes, Paris, Champs Flammarion.

Planck (Max), 1960, Autobiographie scientifique et derniers écrits, trad. par André George, Paris, Albin Michel (Les Savants et le monde).

SoKal (Alan), 1997, Réponse à un article de Bruno Latour, Le Monde, 18 janv.

WeInBERG (Stephen), 1996, Le Rêve d'une théorie ultime, Paris, Odile Jacob. 